\title{
Prueba de calidad de estabilizaciones de suelos
}

\author{
R. SPRINGENSCHMIDT Y H. SOMMER, VIEna \\ Beton, n० 10, octubre 1969, págs. 442.446
}

\section{INTRODUCCION}

Por estabilización del suelo con cemento se entiende, como se sabe, un procedimiento según el cual, de suelos heterogéneos y sueltos puede hacerse un nuevo material, resistente al agua y a las heladas y que, además, acusa una suficiente resistencia a la compresión. Para ello, se mezcla el suelo con el cemento y se le compacta con el contenido de agua óptimo (según Proctor).

Entre estabilización del suelo con cemento y hormigón existen diferencias técnicas fundamentales, por lo cual las pruebas de la calidad también se efectuarán de forma totalmente distinta.

Con hormigón se supone siempre una compactación prácticamente absoluta, de modo que los huecos entre los diferentes granos de grava y arena se hallan casi totalmente rellenos de pasta de conglomerante. Por lo tanto, el determinante de la calidad del hormigón es la pasta de cemento, es decir, la relación agua/cemento.

En estabilizaciones de suelo no se consigue una compactación prácticamente total. A la composición granulométrica del suelo no se le puede exigir aquí nada especial con respecto a un mínimo de huecos. Por consiguiente, quedan en la estructura del grano más huecos que con hormigón, incluso habiendo hecho una buena compactación. Así, por ejemplo, arenas de grano homogéneo, después de la compactación con contenido de agua óptimo todavía tienen inclusiones de aire entre 10 y $15 \%$. En estabilizaciones de suelos se añade también mucho menos cemento (de acuerdo con el suelo, de 80 a $220 \mathrm{~kg}$ por m $\mathrm{m}^{3}$ ), precisamente el suficiente para que el suelo se endurezca convirtiéndose en un material consistente y con la suficiente resistencia a las heladas. En estabilizaciones de suelos el cemento ya no envuelve los granos, sino que los aglutina únicamente en distintos puntos. Incluso con arena gruesa bien escalonada queda todavía del 3 al $5 \%$ de aire ocluido, es decir, más del doble que en el hormigón ( 1 a $2 \%$ ).

En estabilizaciones de suelo, por lo tanto, además de
1) Contenido de agua $y$
2) contenido de cemento,
3) la medida de la compactación 
es determinante de la calidad y, por consiguiente, hay que precisarla siempre. En la prueba de calidad hay que trabajar siempre con un esfuerzo constante de compactación. Las probetas cúbicas, que se confeccionan para el hormigón, no van bien, ya que con mezclas relativamente secas no se consiguen densidades en seco reproducibles. Igual que en mecánica del suelo, para la confección de las probetas se utiliza el aparato de Proctor, con el que se puede mantener un esfuerzo de compactación perfectamente determinado.

La prueba de calidad tiene por objeto:

1. Adquirir la seguridad de que el suelo es adecuado para su estabilización con cemento (estimar su idoneidad puede hacerse previamente).

2. Predecir en la construcción eventuales dificultades que, por ejemplo, tienen influencia en la disposición de la maquinaria.

3. Determinar el contenido necesario de cemento.

4. Determinar el contenido necesario de agua en la compactación.

5. Determinar la densidad mínima.

Condición previa y decisiva para la prueba de calidad es que la muestra que se analiza sea característica del suelo a estabilizar en el lugar de la obra.

Si son de esperar oscilaciones en las propiedades del suelo, hay que tomar varias muestras, acaso una media y dos extremas, y se las analiza por separado. Como quiera que las propiedades del suelo oscilan frecuentemente con más intensidad en el lugar de la obra, es mejor ensayar varias muestras en un análisis sencillo, que con una sola muestra realizar un amplio trabajo de laboratorio. El tamaño de las muestras necesarias para la prueba de calidad se tomará de la tabla 1 .

T A B L A 1

Cantidad de muestra para la prueba de calidad.

\begin{tabular}{|l|c|}
\hline Suelo & Cantidad de muestra mínima \\
\hline Suelos con grava mayor de $20 \mathrm{~mm}$ & $100 \mathrm{~kg}$ \\
Suelo sin grava mayor de $20 \mathrm{~mm}$ & $50 \mathrm{~kg}$ \\
\hline
\end{tabular}

Con el fin de obtener factores comparables internacionalmente, actualmente se realizan esfuerzos tendentes a estudiar un método de ensayo unificado en los países europeos. Este método ha de poder realizarse en forma sencilla, segura y rápida. No obstante, para una prueba sencilla de calidad se necesitan de 2 a 4 semanas (fig. 1). En casos especiales sirven métodos de prueba complementarios. 


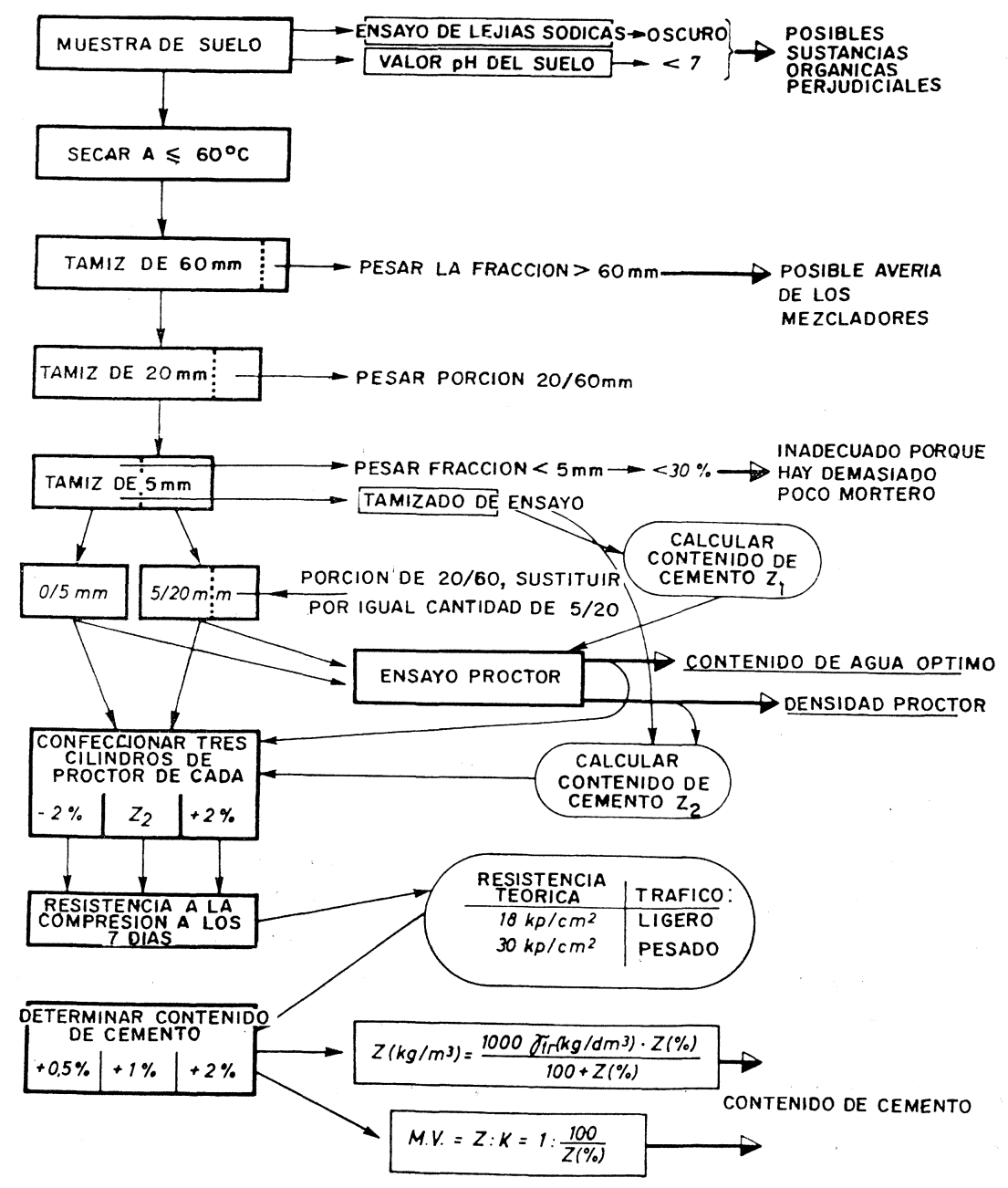

Fig. 1.-Prueba de calidad en suelos granulosos (resumen).

\section{CALIFICACION DE LOS SUELOS}

Idóneos son todos los suelos que mezclados con cemento consiguen una resistencia suficiente. Para la estabilización de la capa portante que se halla sobre la capa protectora contra las heladas y bajo la cubierta o la capa bituminosa de base, son apropiadas prácticamente todas las arenas y arenas gruesas, incluso si contienen hasta $35 \%$ de fracciones cohesivas (= fracciones menores de $0,063 \mathrm{~mm}$ ) o acusan una composición granulométrica desfavorable como son las arenas de granos iguales (arenas volantes).

Claro que son composiciones granulométricas favorables como las conocidas para estabilizaciones mecánicas o en la tecnología del cemento, ya que estos suelos exigen menos cemento. Mejoras en la composición granulométrica sólo se realizan en casos excepcionales y únicamente al mezclar en instalación fija, ya que la mayoría de las veces es necesaria una fase de trabajo complementaria que puede resultar muy cara.

En suelos granulosos (arenas y gravas) hay que hacer tres limitaciones:

1. Que no existan piedras de más de 60 a $100 \mathrm{~mm}$ de diámetro o bien que se eliminen, ya que, en caso contrario, se averiarían los mezcladores. 
2. Que por lo menos el $30 \%$ del suelo tiene que pasar por el tamiz de $5 \mathrm{~mm}$ de malla, a fin de que se forme mortero suficiente y no se produzcan coqueras.

3. Que el suelo no contenga ninguna sustancia orgánica que retarde o impida el endurecimiento del cemento, como es el caso con humus.

Sustancias orgánicas se hallan frecuentemente en capas próximas cercanas a la superficie, y con suelos permeables se encuentran, a veces, en capas más profundas. Las sustancias orgánicas han de ser tenidas en cuenta también al realizar la obra, ya que es posible que sólo aparezcan en algunos puntos y no existan en la muestra de la prueba de calidad.

Suelos coherentes (más de $35 \%$ de fracciones coherentes) se estabilizan como capas portantes para caminos rurales y carreteras comunales, así como en ferrocarriles como remate del nivel del terreno (inmediatamente debajo del balasto de la via). En carreteras con carga mayor, sirven suelos coherentes estabilizados únicamente como remate del nivel de tierra y para el movimiento de las obras. Suelos coherentes son más difíciles de mezclar y exigen más cemento para alcanzar un endurecimiento resistente a las heladas. Por lo tanto, hay que probar siempre si no es más económico en lugar del suelo existente, obtener arena o grava de una cantera cercana y estabilizarla con menos cemento y con mayores rendimientos diarios. Las arcillas con un límite de plasticidad de más de $35 \%$ y una plasticidad de más de $15 \%$ se eliminan normalmente, ya que no se pueden desmenuzar suficientemente con los aparatos usuales en la obra.

En terraplenes o como remate superior del nivel de tierra de carreteras se exige mucho menos en cuanto a la resistencia a la intemperie. A menudo se exige sólo que el nivel no se comprima por el movimiento de la obra y que los trabajos de montaje puedan realizarse también en tiempo lluvioso, de modo que en suelos coherentes pueda encontrarse suficiente resistencia a la intemperie con pequeñas adiciones de cemento.

\section{PRUEBA DE CALIDAD EN SUELOS GRANULOSOS}

\subsection{Componentes orgánicos}

Tan pronto llega la muestra al laboratorio, se toma una pequeña cantidad con la que se efectúa el conocido ensayo con sosa caústica al $3 \%$ (1). Si el líquido resultante después de 24 horas es incoloro o amarillo claro, no es de esperar una influencia perjudicial por parte de los compuestos orgánicos.

Con papel indicador puede determinarse el valor $\mathrm{pH}$ del suelo. Con valores de $\mathrm{pH}$ mayores que 7 son inofensivos la mayoría de las veces los compuestos orgánicos en pequeña proporción, en tanto que con valores de $\mathrm{pH}$ inferiores a 7 pueden ya perjudicar al endurecimiento del cemento, (2). La tabla 2 contiene más indicaciones con respecto a la calidad.

T A в L A 2

Valor del $\mathrm{pH}$ del suelo y compuestos orgánicos -indicaciones con respecto a la calidad.

\begin{tabular}{|l|l|l|}
\hline Valor pH & Color en el ensayo con sosa cáustica & Calidad previsible \\
\cline { 2 - 3 } & Incoloro, amarillo claro o & Buena. \\
$<7$ & amarillo oscuro. & Amarillo oscuro. \\
$<7$ & Rojo-marrón o marrón. & Malemática \\
- & Negro. & Inadecuada. \\
\hline
\end{tabular}


El que realmente no existan en el suelo sustancias orgánicas perjudiciales, no se comprueba con seguridad hasta que tiene lugar el endurecimiento inicial de la mezcla de ensayo con cemento. (En el ensayo de Proctor subsiguiente [véase 3.3] es conveniente levantar la última muestra y al día siguiente se comprueba si se ha endurecido perfectamente). Cemento $\mathrm{Z} 475$ o una adición de cloruro de calcio (aproximadamente 0,6 \% del peso del suelo) puede conducir en suelos con endurecimiento inicial malo a una resistencia suficiente. Como quiera que los componentes orgánicos pueden a menudo estar repartidos irregularmente en el suelo, y por consiguiente crear un gran riesgo en la obra, conviene analizar, en primer lugar, si no se puede estabilizar otro suelo que no contenga ningún compuesto orgánico.

\subsection{Preparación de la muestra y ensayo de tamizado}

Se seca la muestra (temperatura máxima: $+60^{\circ} \mathrm{C}$ ), para que no sean demasiado grandes las diferencias de humedad en el interior de la misma. Entonces es más fácil confeccionar probetas con un determinado contenido de humedad. A continuación se divide la muestra en los grupos granulométricos $0 / 5,5 / 20,20 / 60$ y más de $60 \mathrm{~mm}$, para que en el ensayo de Proctor y en la confección de muestra pueda mantenerse igual relación de grano grueso a grano fino. No es necesario separar cuidadosamente todas las partes finas adheridas a los gránulos de grava, ya que esto tampoco ocurre en la ejecución de la obra. Los granos de más de $20 \mathrm{~mm}$ se tiran normalmente después de la pesada. En una muestra parcial de la proporción $0 / 5 \mathrm{~mm}$ se procede la mayoría de las veces a un tamizado húmedo, con lo que en casos especialmente urgentes sólo se determina la proporción menor de 0,063 mm. En suelos coherentes se determina también, en la fracción menor que 0,04 mm, el límite de líquido y el límite de plasticidad según Atterberg, (3).

\subsection{Ensayo de Proctor}

A continuación se realiza, inmediatamente, el ensayo de Proctor, (3), con el contenido de cemento que probablemente se necesite, y que puede estimarse la mayoría de las veces con suficiente exactitud a base de la clase del suelo (tabla 3 ).

T А B L A 3

Normas para el contenido de cemento necesario.

\begin{tabular}{|l|c|c|c|}
\hline \multicolumn{1}{|c|}{ Clase de suelo } & Necesidad media de cemento & $\begin{array}{c}\text { contenido de cemento para el } \\
\text { ensayo Proctor }\end{array}$ \\
\cline { 2 - 3 } & $(\%)$ & $\left(\mathrm{kg} / \mathrm{m}^{3}\right)$ & $\%$ \\
\hline Grava. & $4-6$ & $80-120$ & 5 \\
Arena, arena limosa. & $6-10$ & $120-160$ & 8 \\
Arena de grano uniforme. & $8-12$ & $140-200$ & 10 \\
Limo. & $7-12$ & $120-180$ & 10 \\
Arcilla. & $9-16$ & $150-220$ & 12 \\
\hline
\end{tabular}

De acuerdo con la experiencia, aumentando el contenido de cemento, de, por ejemplo, 6 al $8 \%$, casi no varía en la mayoría de los suelos el contenido de agua óptima, y la densidad 
Proctor sólo varía ligeramente. El contenido de cemento se expresa en partes en peso referidas a 100 partes de suelo seco. En la práctica estas partes en peso se expresan como tanto por ciento.

Para el recipiente Proctor de $10 \mathrm{~cm}$ de diámetro no ha de tener la muestra ningún grano mayor de $20 \mathrm{~mm}$, ya que estos granos falsearían el resultado. No se tienen en cuenta las piedras mayores de $60 \mathrm{~mm}$, ya que están repartidas irregularmente en la obra. La proporción de grava entre 20 y $60 \mathrm{~mm}$ se sustituye por una cantidad en peso igual de grava de $5 / 20 \mathrm{~mm}$. Se obtiene así la misma densidad aproximadamente que en el ensayo con el material primitivo en un recipiente de $30 \mathrm{~cm}$ de diámetro, (4).

Primeramente hay que determinar los contenidos de agua de las proporciones de $0 / 5 \mathrm{y}$ $5 / 20 \mathrm{~mm}$ por secado en horno de muestras suficientemente grandes según la tabla 4 , y en el cálculo tener en cuenta las cantidades necesarias secadas al aire.

T A в L A 4

Cantidad de muestra para la determinación del contenido de agua (*).

\begin{tabular}{|c|c|}
\hline Grano máximo de la muestra & Peso en húmedo aproximado \\
$(\mathrm{mm})$ & $(\mathrm{g})$ \\
\hline 2 & 100 \\
5 & 300 \\
20 & 750 \\
60 & 3.000 \\
\hline
\end{tabular}

(*) Estas cantidades de muestra son en parte mayores que las indicadas en (3), porque según la experiencia en el cilindro Proctor es donde el agua se distribuye más irregularmente.

Generalmente, en el ensayo Proctor se compacta varias veces la misma muestra con contenido de agua creciente, sin intercalar ninguna pausa de trabajo. En suelos sin gravas de más de $5 \mathrm{~mm}$, pesa esta muestra corrientemente $3.500 \mathrm{~g}$. Si el suelo contiene grano más grueso, se necesitan $5.500 \mathrm{~g}$. Si existen granos quebradizos que quedan destruidos al estamparlos, para cada ensayo parcial hay que tomar una nueva muestra del suelo de 2.400 ó 3.300 g cada una. Para determinar el contenido de agua se toma del centro de cada cuerpo Proctor una muestra parcial (según tabla 4) y se la seca en estufa. En el último ensayo parcial ha de conservarse el cilindro de la muestra, para poder comprobar al día siguiente si se produce un endurecimiento normal. Por eso se toma la muestra de contenido de agua después de introducir la segunda capa de la mezcla restante.

Para la ejecución de la obra suministra el ensayo Proctor el contenido de agua que se persigue, el cual se indica como en la mecánica del suelo en partes en peso referidas a 100 partes de peso de sustancias sólidas o bien en por ciento, con la diferencia de que aquí se cuenta entre las sustancias sólidas no solamente el suelo, sino también el cemento considerado en estado anhidro. En suelos granulosos se queda, en la ejecución de la obra, la mayoría de las veces, escaso en el contenido de agua óptimo; en suelos coherentes el sobrepasar el contenido de agua óptimo es favorable para la resistencia a las heladas, siem- 
pre que el suelo se deje compactar perfectamente. La mayor densidad en seco (densidad Proctor) que puede alcanzarse en el ensayo Proctor hay que perseguirla también en la ejecución de la obra, en el supuesto de que se confirme el resultado del ensayo Proctor en la subsiguiente confección del cilindro de ensayo.

\subsection{Confección de cilindros de ensayo}

A base de la densidad Proctor y de las proporciones de más de 5 y de menos de 0,063 mm puede estimarse más exactamente el contenido de cemento necesario. Para esto sirve un diagrama que se creó a base del análisis de varios miles de muestras de suelos (fig. 2).

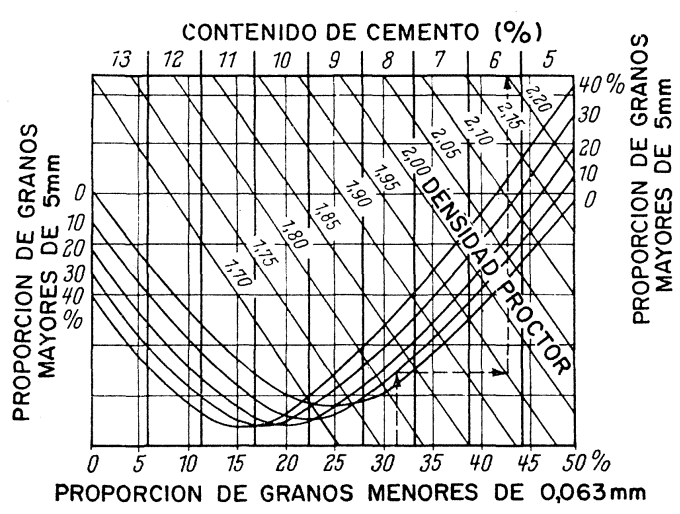

Fig. 2.-Diagrama para calcular el contenido de cemento necesario, (4).

Con el contenido de cemento tomado de la tabla se confeccionan los cilindros de ensayo. Si se aspira a una resistencia a 7 días de unos $18 \mathrm{kp} / \mathrm{cm}^{2}$, se preparan además cilindros con un contenido de cemento $2 \%$ menor y $2 \%$ mayor. Si se exigen resistencias a 7 días de 30 a $40 \mathrm{kp} / \mathrm{cm}^{2}$, sirve como límite inferior el contenido de cemento tomado de la figura 2 . Los demás cilindros de ensayo contienen proporciones de cemento 2 y $4 \%$ mayores.

Con los tres contenidos de cemento distintos y el contenido de agua óptimo se confeccionan tres cilindros de ensayo para cada caso. Para cada cilindro se hace la mezcla por separado con el fin de poder mezclar más cuidadosamente y evitar segregaciones en la composición. Se necesitan cantidades de suelo de $2.400 \mathrm{~g}$ o, en suelos con granos de más

$5 \mathrm{~mm}$, de $3.300 \mathrm{~g}$. Primeramente se mezcla a mano el suelo secado al aire de $0 / 5 \mathrm{~mm}$ y cemento seco, añadiéndole a continuación la cantidad de agua necesaria hasta que se produce una coloración uniforme. Entonces se le agrega mezclando la parte de suelo de 5/20 milímetros. En suelos coherentes se agrega ya el agua el día anterior y durante la noche se le deja penetrar en la muestra, con el fin de que los terrones de tierra estén bien humedecidos en su interior y al entrar agua posteriormente no se creen fuerzas de hinchamiento elevadas (véase 4.3).

Las mezclas se compactan como en el ensayo Proctor, pero con las siguientes diferencias:

- Antes de introducir la segunda y tercera capa hay que hacer rugosa la capa anterior, con el fin de que se adhieran bien entre sí. Para ello, con un cuchillo o destornillador se hacen en la superficie estrías de varios $\mathrm{mm}$ de profundidad.

- En suelos pedregosos, después de verter la mezcla, hay que pinchar hacia abajo en las paredes del cilindro con un cuchillo largo o una espátula estrecha, con el fin de que no se formen coqueras.

- Una vez introducida la segunda capa, del resto de la mezcla se toma una muestra parcial para determinar el contenido de agua (véase tabla 4).

Después de raseada la superficie se pesan los cilindros con el recipiente y a continuación se prensan, desmoldándose en estado fresco (fig. 3). Esto se hace fácilmente en muchos 
suelos, si el recipiente Proctor es ligeramente cónico, de modo que su diámetro inferior sea aproximadamente $0,4 \mathrm{~mm}$ mayor que el superior. No obstante, para el prensado se aconseja utilizar un gato de coche, porque el prensado a mano crea unas sacudidas y se estropea fácilmente el cilindro de ensayo. En cilindros que se deforman al agarrarlos o se desmoronan completamente, antes del prensado se coloca debajo un disco de celuloide de unos $0,2 \mathrm{~mm}$ de grueso y $9,9 \mathrm{~cm}$ de diámetro y con una espátula se levanta la base del cuerpo. Los cilindros se llevan así con la superficie raseada sobre una base plana, por ejemplo una placa de vidrio. La mayoría de las veces ya no es necesario un raseado de las superficies de compresión con el mortero de cemento o yeso.

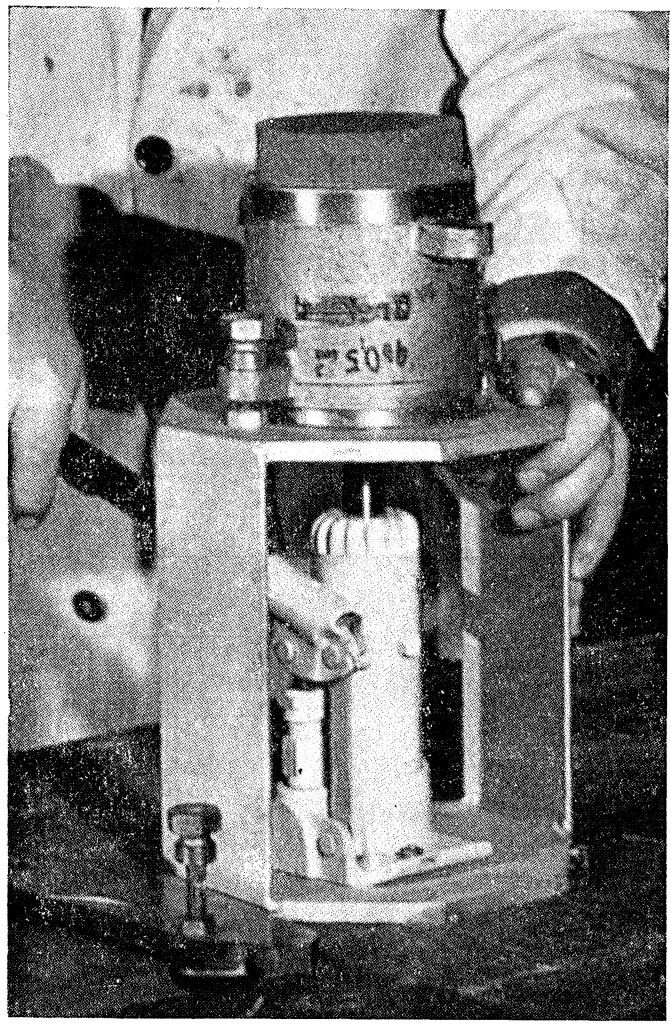

Fig. 3.-Prensado de un cilindro Proctor.

La dispersión de la densidad Proctor no debe sobrepasar los $0,05 \mathrm{~kg} / \mathrm{dm}^{3}$ y la del contenido de agua óptimo el $1 \%$.

Generalmente se almacenan los cilindros de ensayo 7 días en cámara húmeda a $+20^{\circ} \mathrm{C}$, y antes del ensayo de resistencia a la compresión se colocan 4 horas bajo agua. Una permanencia en agua de más de 24 horas sólo tiene una influencia muy reducida en suelos impermeables, pero tiene el inconveniente técnico de que, por ejemplo, para ensayos en un lunes hay que empezar el domingo anterior la permanencia en el agua.

A veces se exigen también resistencias a los 28 días. De la resistencia a los 7 días se puede deducir normalmente la resistencia a los 28 días y no se cometen grandes errores. Pero en ningún caso debe de renunciarse a una determinación de la resistencia a los 7 días, porque sustancias orgánicas perjudiciales, a menudo, no se reconocen ya a los 28 días.

\subsection{Elección del contenido de cemento}

La pregunta de a qué resistencia se ha de aspirar, se discute con mucha frecuencia.

Según la experiencia, conglomerantes pulverulentos como el cemento pueden mezclarse en cantidades aumentadas, al contrario de lo que ocurre con sustancias líquidas, sin que se creen dificultades en la ejecución de la obra. Pero, llega un límite para el que no aumenta más el valor de utilización de la capa estabilizada. Para hacer que un suelo se endurezca hasta formar una sustancia sólida y para evitar que se reblandezca por agua, heladas o cargas móviles, bastan resistencias relativamente bajas. La figura 4 muestra el aumento de la capacidad portante de una capa estabilizada con contenido de cemento creciente. Tan pronto es suficiente el contenido de cemento para endurecer el suelo de manera que sea resistente a las heladas, no aumenta más la capacidad portante. Unicamente con mayores espesores de capa se obtiene una capacidad resistente mayor, pero no por un mayor contenido de cemento.

La resistencia a la helada es, pues, decisiva para determinar el contenido de cemento, 
no influyendo tanto en la resistencia a la compresión. Pero para arenas y gravas, a partir de una resistencia a la compresión de unos $18 \mathrm{kp} / \mathrm{cm}^{2}$ se puede estar seguro, después de 7 días, de que el suelo se ha endurecido a prueba de heladas, (4).

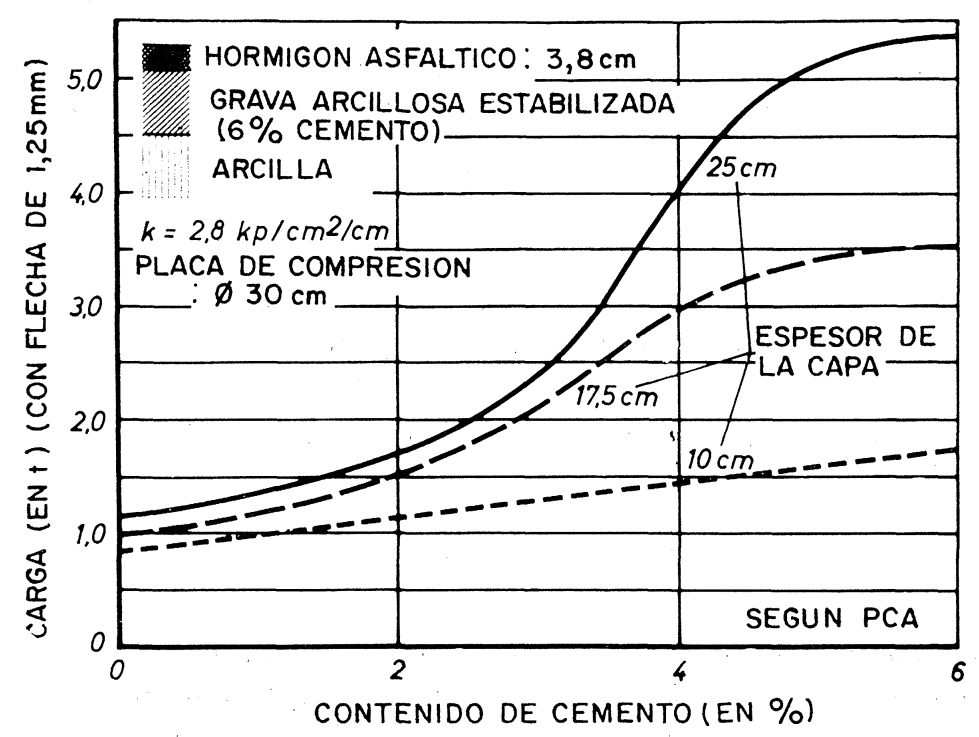

Fig. 4.- Influencia del contenido de cemento en la capacidad resistente de grava arcillosa estabilizante (con $6 \%$ de cemento resistente a las heladas), (5).

Por lo tanto, en lugar de los costosos ensayos de resistencia a las heladas se emplea, actualmente, la resisteńcia a la compresión, mucho más fácil de realizar, no siendo esta resistencia más que un índice de calidad fácil de comprobar sin importancia directa para el valor de utilización de la capa estabilizada.

Es esencial el que se haya alcanzado con seguridad la resistencia a las heladas y un endurecimiento permanente, a pesar de las mayores irregularidades naturales de la estabilización del suelo.

Si se pudiera hacer una estabilización de cemento con resistencia uniforme, es decir, sin dispersiones, bastaría en la carretera, según el tipo de subsuelo, movimiento de las obras e influencia de las heladas, una resistencia a la compresión de unos 10 a $15 \mathrm{kp} / \mathrm{cm}^{2}$ después de 7 días. Claro es que hay que prever seguridades para que la capa estabilizada se endurezca perfectamente en toda su extensión. Esta seguridad ha de ser mayor en carreteras de primer orden $\mathrm{y}$, en este caso, se exigen frecuentemente resistencias a la compresión a los 7 días de $30 \mathrm{kp} / \mathrm{cm}^{2}$, lo que conduce a resistencias a los 28 días de unos 50 kilopondios $/ \mathrm{cm}^{2}$. En carreteras secundarias y caminos son más bajas las resistencias y el contenido de cemento, con el fin de que las grietas de enfriamiento no penetren tan fácilmente por la delgada capa bituminosa. En este caso se han generalizado resistencias a 7 días de $18 \mathrm{kp} / \mathrm{cm}^{2}$, correspondientes a una resistencia a 28 días de unos 30 kilopondios $/ \mathrm{cm}^{2}$.

Si hay que alcanzar resistencias de $30 \mathrm{kp} / \mathrm{cm}^{2}$ después de 7 días, por ejemplo en la estabilización de capas protectoras contra heladas de autopistas, se toma entonces el contenido del cemento con el que los cilindros de ensayo han dado esta resistencia y se le aumenta 
en una determinada medida de reserva, a fin de considerar la peor mezcla en obra. Si se trabaja con mezcladoras de varios pasos, es aconsejable una medida de reserva de aproximadamente $2 \%$; con mezcladoras de 1 paso, basta a menudo $1 \%$; y en mezcladoras con tiempo de mezclado lo suficientemente largo y dosificación exacta, puede ser suficiente un aumento de $0,5 \%$ de cemento.

El último proceso de la prueba de calidad es, en resumidas cuentas, la conversión del contenido de cemento en tanto por ciento a $\mathrm{kg}$ por $\mathrm{m}^{3}$ de mezcla compactada según:

$$
\mathrm{Z}\left(\mathrm{kg} / \mathrm{m}^{3}\right)=\frac{1.000 \cdot \gamma_{\mathrm{tr}}}{\left.100+\mathrm{kg} / \mathrm{dm}^{3}\right) \cdot \mathrm{Z}(\%)} ;
$$

en la cual:

$$
\gamma_{\mathrm{tr}} \text { es la densidad Proctor }\left(\mathrm{en} \mathrm{kg} / \mathrm{dm}^{3}\right. \text { ). }
$$

Para mezcladoras fijas hay que indicar la relación de mezcla M. V. según:

$$
\text { M.V. }=\mathrm{Z}: \mathrm{K}=1: \mathrm{k}=1: \frac{100}{\mathrm{Z}(\%)}
$$

El contenido de cemento ha de sèr en obra, con mezcladoras de varios pasos, por lo menos de $105 \mathrm{~kg} / \mathrm{m}^{3}$; en mezcladoras de un paso, por lo menos de $100 \mathrm{~kg} / \mathrm{m}^{3}$; y en mezcladoras forzadas fijas, como mínimo de $80 \mathrm{~kg} / \mathrm{m}^{3}$, ya que, en caso contrario, no se garantiza, in situ, una mezcla uniforme.

\section{PRUEBA DE CALIDAD EN SUELOS COHERENTES}

En suelos coherentes y en todos los suelos granulares en los cuales existan temores con respecto a la resistencia a las heladas - acaso debido a grano grueso muy quebradizo, poroso o expuesto a la intemperie-, son necesarios los ensayos de heladicidad.

Hay que tener en cuenta que la seguridad a prueba de heladas de un suelo no tiene nada que ver directamente con el endurecimiento a prueba de heladas de una estabilización. $\mathrm{Si}$ un suelo coherente que se mueve mucho por las heladas, es decir, que forma lagunas de hielo, se estabiliza correctamente, por la acción de las heladas, se mantiene como una sustancia sólida y no forma lagunas de hielo en el futuro. $\mathrm{Si}$, por ejemplo, una grava que como suelo es resistente a las heladas, se mezcla sólo con $2 \%$ de cemento o se le compacta mal, las heladas pueden producir esponjamientos, de forma que de un suelo estabilizado resulta nuevamente grava.

\subsection{Elevaciones o esponjamientos por heladas}

Si sólo se trata de evitar elevaciones por el hielo, la formación de lentejones de hielo se reproduce en el laboratorio mediante un ensayo sencillo, en el cual se congela desde arriba el cilindro de ensayo, manteniendo su parte inferior sumergida en un baño de agua que se mantiene a $+4^{\circ} \mathrm{C},(6)$. Pequeñas adiciones de cemento impiden ya que se formen lentejones de hielo (fig. 5). Aquí desempeña un papel grande el contenido de agua. Cuanto más mojado se halla el suelo en la compactación, tanto menos cemento se necesita, (7).

\subsection{Reblandecimientos superficiales}

Con contenidos de cemento que no permiten una formación de lentejones o lagunas de hie- 
lo, las heladas pueden, sin embargo, producir aflojamientos, los cuales empiezan como reblandecimientos superficiales y se van profundizando rápidamente, sin que con ello se formen lentejones de hielo. Con el fin de evitar esto, hay que probar con qué contenido de cemento una acción reiterada de las heladas no puede aflojar más la estructura del suelo estabilizado.

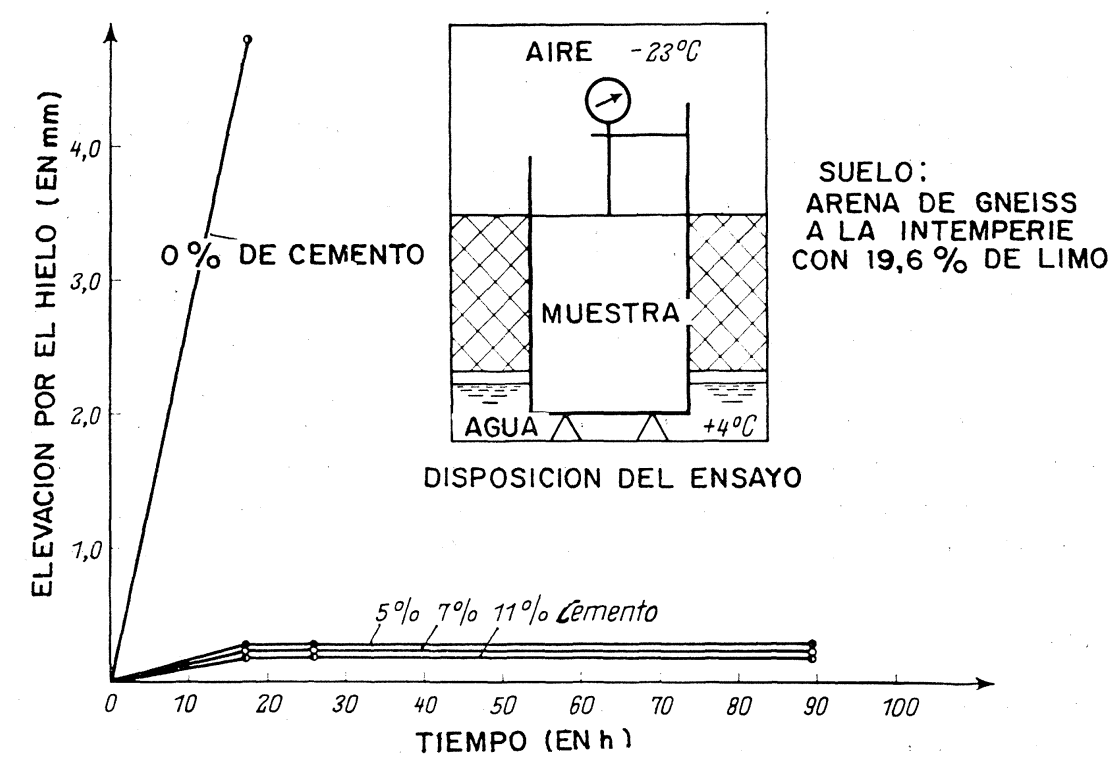

Fig. 5.-Elevaciones o esponjamientos por heladas en un suelo estabilizado, (6).

Desde 1935 se prueba en USA la resistencia a las heladas por medio de un ensayo en el que los cilindros, después de 7 días de endurecimiento, colocados sobre fieltro mojado, se congelan 12 veces cada 24 horas en aire a $-23^{\circ} \mathrm{C}$ y a continuación vuelven a descongelarse durante 24 horas en el recinto húmedo $\mathrm{a}+21^{\circ} \mathrm{C}$. Este ensayo se describe en la norma ASTM D 560-57, (8), y se reproduce brevemente en (9) y (10). Al principio, los cilindros se cepillaban después de cada período de descongelación para eliminar las partículas superficiales sueltas, prescribiéndose que durante los 12 cambios de congelación-descongelación, y de acuerdo con la clase del suelo, sólo debe de perderse, como máximo, 7 al $14 \%$ del peso, (9). Hoy en día, se emplea un criterio más sencillo: no se cepillan los cilindros, sino que se mide su longitud después de la primera y después de ia duodécima congelación (fig. 6). Si el alargamiento en dirección longitudinal no sobrepasa el $1 \%$, es decir, $0,12 \mathrm{~mm}$, es suficiente el contenido de cemento, (11).

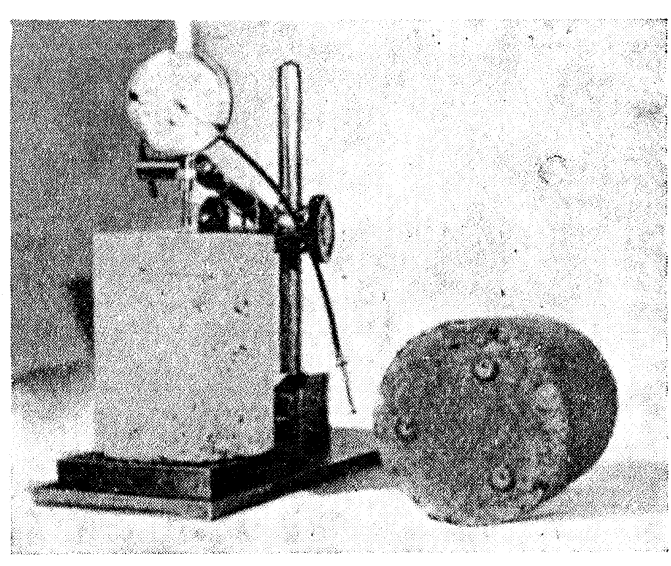

Fig. 6.-Medición de variaciones longitudinales en el ensayo de ciclos de hielo-deshielo. 
Para el ensayo en los ciclos de hielo-deshielo, basta una probeta por cada contenido de cemento. Los índices de medición se adhieren con rexina epoxi rebajada con polvo de piedra o fibras de amianto hasta formar un caldo espeso. Y siendo el cilindro de prueba de 3 ó 4 días, se le fijan por ejemplo, sobre una superficie de base, como puntos de medición, tres arandelas suplementarias de latón, y al día siguiente, sobre la otra superficie de base, un remache de cobre. Los cuerpos se hallan aquí sobre fieltro mojado únicamente durante los períodos de descongelación, pero no durante los períodos de congelación, ya que, caso contrario, se adherirían con el frío y no podrían ser medidos.

Muchos suelos coherentes dan, con el contenido de cemento suficiente para un endurecimiento resistente a las heladas, una resistencia a 7 días que se encuentra por debajo de $18 \mathrm{kp} / \mathrm{cm}^{2}$. Para resistencias mayores se necesita mucho más cemento, sin que aumente el factor de utilización de la capa final (véase 3.5). En algunos limos de baja formabilidad, los cuales son muy adecuados para la estabilización, no hay modo de conseguir las resistencias de 7 ó 28 días prescritas para suelos granulosos, ya que estos suelos endurecen lentamente al principio. Mayores contenidos de cemento no dan en estos suelos un aumento de resistencia hasta después de meses, (12). Por lo tanto, en suelos coherentes se ha de exigir únicamente la resistencia a las heladas y no se prescribirá adicionalmente una determinada resistencia a la compresión. Se indica sólo la resistencia para el contenido de cemento necesario después de los ensayos de cliclos de congelación y descongelación, ya que esto puede ser necesario para el control de la ejecución de la obra, (13).

\subsection{Trituración}

En suelos coherentes surge la cuestión de a qué tamaño hay que desmenuzar los diversos terrones del suelo. Aquí hay que tener cuidado, ya que el grado de trituración puede alterar muchísimo el resultado del ensayo en el laboratorio y la calidad en la obra. Cuanto mejor se tritura, tanto mayores son la consistencia a las heladas y la resistencia.

Normalmente se prescribe que en el lugar de la obra ha de pasar por la criba de $5 \mathrm{~mm}$, por lo menos el $80 \%$ de la mezcla suelo-cemento, escogiéndose aquí granos de grava que queden sobre la criba y que no cuentan. También en la prueba de calidad se aspira a un paso de $80 \%$. Después del mezclado no deben existir ya trozos de material conglomerado mayores de $20 \mathrm{~mm}$. Además, todos los trozos coherentes han de estar húmedos interiormente (véase 3.4 ).

\section{RESUMEN}

En la prueba de calidad se determina:

el contenido óptimo de agua con el que ha de compactarse en el lugar de la obra;

la densidad Proctor que ha de alcanzarse aquí;

el contenido de cemento con el que la capa estabilizada se endurece con resistencia a las heladas.

Los cilindros de ensayo $(\mathrm{h}=12 \mathrm{~cm}, \mathrm{~d}=10 \mathrm{~cm})$ de mezcla de suelo-cemento se compactan con el contenido óptimo de agua como en el ensayo Proctor y se los almacena húmedos durante 7 días. Antes del ensayo de resistencia a la compresión se los mantiene durante 4 horas bajo agua. Suelos granulosos estabilizados con cemento son resistentes a las heladas cuando la resistencia a la compresión a los 7 días es de $18 \mathrm{kp} / \mathrm{cm}^{2}$. En carreteras para tráfico pesado se determina el contenido de cemento necesario con arreglo a una mayor 
resistencia a los 7 días, de unos $30 \mathrm{kp} / \mathrm{cm}^{2}$, a pesar de que, por ello, la capacidad portante de la capa estabilizada ya no aumenta más en forma perceptible; sin embargo, se aspira a una mayor seguridad contra las irregularidades en la ejecución de la obra. Con el fin de compensar la peor mezcla en obra, de acuerdo con la calidad de la mezcla, se aumenta el contenido de cemento de 0,05 a $2,0 \%$.

En suelos coherentes y todos los suelos con granos quebradizos, porosos o blandos, se ensayará directamente la resistencia a las heladas. Las probetas se someten a 12 ciclos de hielo-deshielo según ASTM D 560-57. Pero ya no se cepillan más los cilindros, sino que se mide su longitud después de la primera congelación y después de la duodécima. El contenido de cemento es suficiente si el alargamiento no sobrepasa $1 \%$.

\section{BIBLIOGRAFIA}

(1) Vorläufiges Merkblatt für die Bestimmung der organischen Bestandteile im Boden. Forschungsgesellschaft für das Straßenwesen e. V., Köln 1965

(2) Sherwood, P. T.: The Effect of Soil Organlc Matter on the Setting of Soil-Cement Mixtures. Road Research Technical Paper No. 61, Her Majesty's Stationery Office, London 1962.

(3) Merkblatt für bodenphysikalische Prüfverfahren im Straßenbau. Forschungsgesellschaft für das Straßenwesen im Osterreichischen Ingenieur- und Architekten-Verein, Wien 1963, und.

Forschungsgesellschaft für das Straßenwesen e. V., Köln 1963.

(4) Norling, L. T., and Packard, R. G.: Expanded Short-Cut Test Method for Determining $\mathrm{C}_{\theta}$ ment Factors for Sandy Solls. Bull. 198. Highway Research Board, Washington 1958.

(5) Sluis, F. A. van der: Grond-cement in de Vereenigde Staten von Noord-Amerika. Stlchting Studie-Centrum Wegenbouw, Arnhem 1961.

(6) Springenschmid, R.: Zur Technologie und Praxis der Bodenverfestigung mit Zement. Straßenbau-Technik 16 (1963) H. 4.
(7) Springenschmid, R.: Der Einfluß des Elnbauwassergehaltes auf das Frostverhalten verdichteter und stabilisierter Böden. Konferenzberichte der Internationalen Diskussionstagung über Bodenmechanik im Straßenbau. Osterreichischer Ingenieur- und ArchitektenVerein, Wien 1964, und

Felsmechanik und Ingenieurgeologie Vol. III/3-4, Wien 1965.

(8) ASTM Designation D 560-57. 1961 Book of ASTM Standards, Part 4, S 1349-1355, American Society for Testing and Materials, Philadelphia 1961.

(9) Soil-Cement Laboratory Handbook. Portland Cement Association, Chicago 1959 (4th edition). Deutsche Ubersetzung Fachverband Zement e. V., Köln 1968.

(10) Vorläufiges Merkblatt für Eignungsprüfungen bei Bodenverfestigungen mit Zement. Forschungsgesellschaft für das Straßenwesen e. V., Köln 1959.

(11) Packard, R. G., and Chapman, G. A.: Developments in Durability Testing of Soil-Cement-Mixtures. Record 36, Highway Research Board, Washington 1963.

(12) Springenschmid, R., und Helms-Derfert, H.: Einfluß des Zementes aul die Eigenschaften von Boden-Zement-Gemischen. beion 13 (1963) H. 12.

(13) Technische Vorschriften und Richtlinien für die Ausführung der Tragschichten unter Fahrbahndecken (T. V. T.). Entwurf für Abschnitte 2-7. Fassung Juli 1967. Forschungsgesellschaft für das Straßenwesen e. V., Köln 1968. 